\title{
Polymorphic Microsatellite Marker
}

National Cancer Institute

\section{Source}

National Cancer Institute. Polymorphic Microsatellite Marker. NCI Thesaurus. Code C18299.

Short repeats of nucleotide sequences present throughout the genome which exhibit variations within a population. 